Philippe Block · Jan Knippers

Niloy J. Mitra · Wenping Wang Editors

Advances in

Architectural

Geometry 2014

算 Springer 


\title{
Interlocking Folded Plate: Integrated Mechanical Attachment for Structural Wood Panels
}

\author{
Christopher Robeller, Andrea Stitic, Paul Mayencourt, and Yves Weinand
}

\begin{abstract}
Automatic joinery has become a common technique for the jointing of beams in timber framing and roofing. It has revived traditional, integrated joints such as mortise and tenon connections. Similarly, but only recently, the automatic fabrication of traditional cabinetmaking joints has been introduced for the assembly of timber panel shell structures. First prototypes have used such integrated joints for the alignment and assembly of components, while additional adhesive bonding was used for the load-bearing connection. However, glued joints cannot be assembled on site, which results in several design constraints.

In this paper, we propose the use of dovetail joints without adhesive bonding, on the case study of a timber folded plate structure. Through their single-degree-offreedom (1DOF) geometry, these joints block the relative movement of two parts in all but one direction. This presents the opportunity for an interlocking connection of plates, as well as a challenge for the assembly of folded plate shells, where multiple non-parallel edges per plate must be jointed simultaneously.
\end{abstract}

\section{Introduction}

Architectural designs have often been inspired by folded shapes such as origami, however the folding principle can rarely be applied to building structures directly. Alternatively, folded plates can be cast as concrete thin-shells (Trautz and Herkrath 2009), which requires complex formwork and an elaborate vertical support structure. Constructions with discrete elements have been realized with fiber-reinforced plastics (Pizzi 2003) or tubular steel.

A prefabricated folded plate built from cross-laminated timber panels has been proposed by Buri (2010). It combines the elegant and efficient shape of folded plate shells with the advantages of structural timber panels, such as $\mathrm{CO}_{2}$ storage and a low weight-to-strength ratio. However, a major challenge in the design of a

\footnotetext{
C. Robeller $(\varangle) \bullet$ A. Stitic $\bullet$ P. Mayencourt $\bullet$ Y. Weinand

EPFL Timber Construction Laboratory IBOIS, Station 18, CH 1015 Lausanne, Switzerland e-mail: christopher.robeller@epfl.ch; andrea.stitic@epfl.ch; paul.mayencourt@epfl.ch;

yves.weinand@epfl.ch
} 
timber folded plate is presented by the joints: Since timber panels cannot be folded, a large amount of edgewise joints has to provide two main functions. One of these functions is the load-bearing behaviour, where Connector features of the joints must provide a sufficient stiffness and rigidity. The second main function of the joints is the assembly of the parts, where Locator features of the joints are essential for a precise and fast positioning and alignment of the parts.

Hahn (2009) examined the structural behaviour of a first timber folded plate shell built from plywood and assembled with screwed miter joints, concluding that the load-bearing performance could be improved significantly with more resistant connections.

Inspiration for such improvements may be found in integral mechanical attachment techniques, the oldest known technique for the jointing of parts, where the geometry of the parts themselves blocks their relative movements (Messler 2006). Such integrated joints have recently been re-discovered by the timber construction industry. Beginning in 1985, mortise-and tenon joints have been repatriated in timberframe and roof constructions (Hundegger 2014). Only very recently, integrated joints have also been proposed for the edgewise jointing of timber panels. La Magna et al. (2013) and Krieg et al. (2014) have applied finger joints to plywood panels and Robeller et al. (2014b) have demonstrated an application of dovetail joints for cross-laminated timber panels (CLT). In these prototype structures, the integrated joints have played an important role for the assembly of the components. They have also participated in the load-bearing connection of the parts, but additional adhesive bonding was needed. With few exceptions (HESS 2014), such glued joints cannot be assembled on site, because they require a curing period with a specific temperature and humidity (Purbond 2011). Therefore, their application is limited to off-site assembly of larger components, which complicates both transport and handling while still requiring additional connectors for the final assembly.

In this paper, we propose the use of dovetail joints without additional adhesive bonding, on the case study of a timber folded plate shell (Fig. 1).
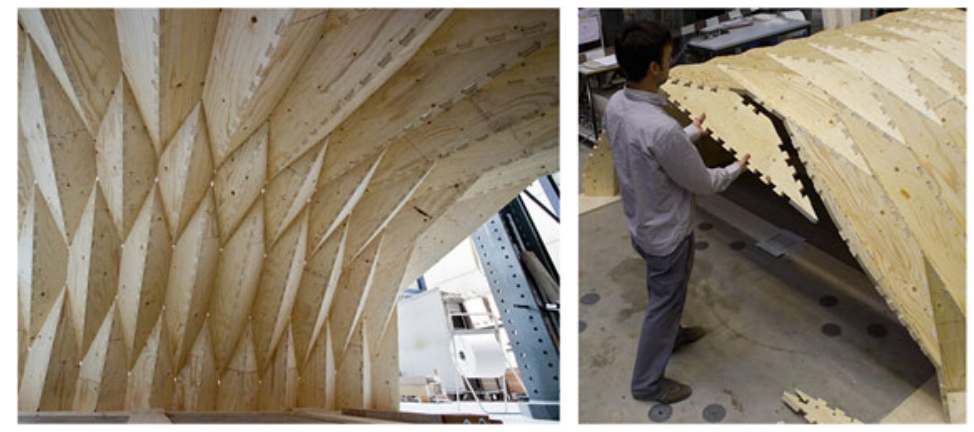

Fig. 1 Timber folded plate built from $21 \mathrm{~mm}$ LVL panels, assembled with single-degree-offreedom dovetail joints without adhesive bonding. Components interlock with one another 
Through their single-degree-of-freedom(1DOF) geometry, these joints block the relative movement of two parts in all but one direction. This presents the opportunity for an interlocking connection of plates, as well as a challenge for the assembly of folded plate shells, where multiple non-parallel edges per plate must be jointed simultaneously.

\subsection{Dovetail Joint Geometry and Mechanical Performance}

Using polygon mesh processing, we describe an edgewise joint based on its edge $E$. From the mesh connectivity, we obtain the edge vertices $p$ and $q$ and the adjacent faces $F_{0}$ and $F_{1}$ with their face normals $n_{0}, n_{1}$. We use the polygon mesh to represent the mid-layer of timber panels with a thickness $t$ and offset $F_{1}$ and $F_{2}$ at $\pm \frac{t}{2}$ to obtain the lines $L$ (Fig. 2a). From a division of $E$, we obtain the points $X_{j}$ for a set of reference frames $\left\{u_{1}, u_{2}, u_{3}\right\}$, where $u_{1} \| \overrightarrow{p q}$ and $u_{2} \| n_{0}$ (Fig. 2b). A finger joint geometry is obtained from an intersection of planes located at $X_{j}$, normal to $u_{1}$, with the four lines $L$.

Without additional connectors, finger joints are planar joints with three degrees of freedom (3DOF). They can resist shear forces parallel to the edge and inplane compressive forces. However depending on the plate geometry, thickness and most of all rotational stiffness of the connection detail, bending moments are also transferred between the plates. Also, due to the rotation of the plate edge caused by bending, in-plane traction forces perpendicular to the edge line appear and their magnitude increases under asymmetrical loads. Such forces, which occur as a result of out-of-plane loading, cannot be supported only by shear and in-plane compression resistant joints (Fig. 3).

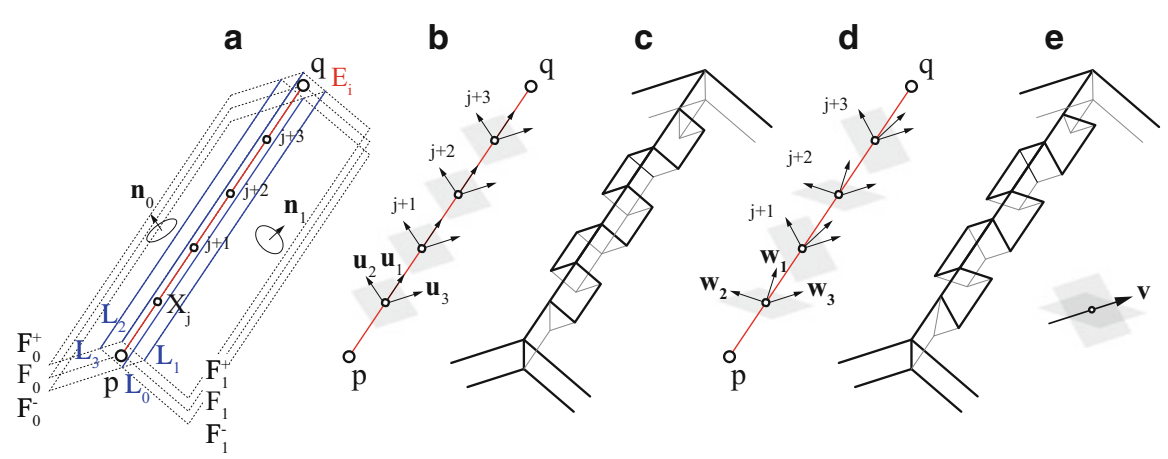

Fig. 2 Joint geometry. (a) Basic parameters, (b) Intersection planes (grey) normal to $\overrightarrow{p q}$, (c) 3DOF joint, (d) Rotated intersection planes (grey) normal to $\vec{w}_{j}$, (e) $1 \mathrm{DOF}$ joint 

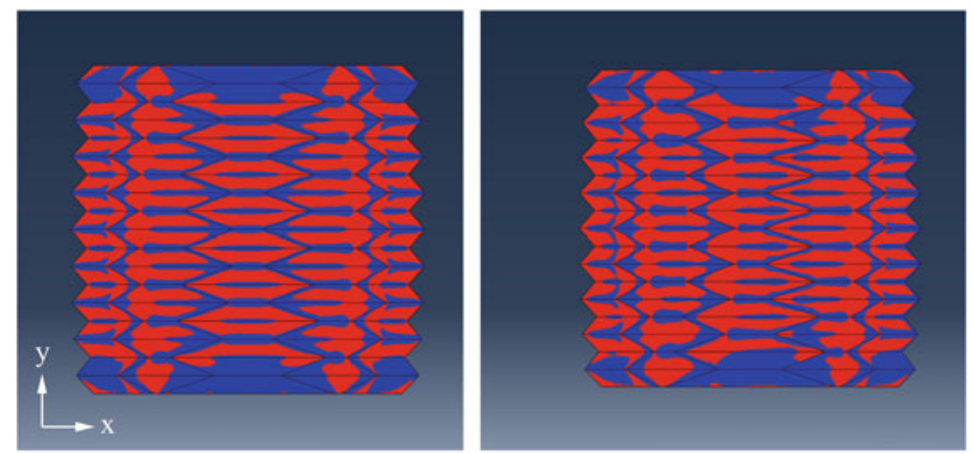

Fig. 3 FEM analysis (top view) of a $3 \times 3 \mathrm{~m}, 21 \mathrm{~mm}$ Kerto-Q folded plate thin shell assuming fully stiff joints. Distribution of traction (red) and compression (blue) stresses in the y direction. Left side: gravity load case. Right side: asymmetric snow load

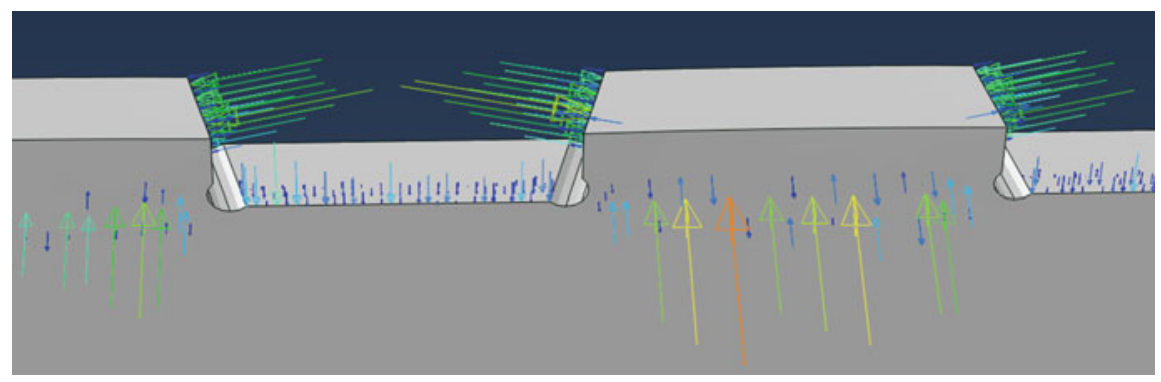

Fig. 4 FEM simulation of bending on a dovetail joint connecting two Kerto-Q $21 \mathrm{~mm}$ LVL panels. The bending moment applied is transformed into compression, normal and shear forces parallel to the inclined contact faces

On a dovetail joint (Fig. $2 \mathrm{~d}$, e), the intersection planes on the points $X_{j}$ are normal to a rotated vector $w_{1}$. It is obtained from a rotation of the reference frame $\left\{u_{1}, u_{2}, u_{3}\right\}$ about $u_{3}$ at an alternating angle $\pm \theta_{3}$. The resulting rotated side faces reduce the dovetail joints degrees of freedom to one translation $\vec{w}_{3}$ (1DOF). Sebera and Simek (2010) have suggested $\theta_{3}=15^{\circ}$ for spruce plywood panels. Such prismatic joints can only be assembled or disassembled along one assembly direction $\vec{v}=\vec{w}_{3}$. In addition to the finger joints resistance to shear and compressive forces, dovetail joints can, without adhesive bonding, also resist bending moments and traction forces which are not parallel to $\vec{v}$. Due to the inclination of the side faces of the joint, resistance to these forces can be improved significantly. In that way the inclined faces take over the role that the glue would have in a finger joint (Fig. 4). 


\subsection{Fabrication Constraints}

One of the main reasons for the resurgence of finger and dovetail joints is the possibility of automatic fabrication. However, the mechanical performance of the joints depends on fabrication precision. At the same time, fast machine feed rates are important for a time-efficient production. We have fabricated such joints with a robot router and a gantry router, achieving higher precision with the gantry machine, which is more stiff and provides a higher repeat accuracy.

The variability of the machine-fabricated joints is enabled by the 5-axis capability of modern routers: Although traditional edgewise joints in cabinetmaking were used for orthogonal assemblies, both the finger and dovetail joint can also be applied for non-orthogonal fold angles, which was essential for the reference projects mentioned before. However, there are certain fabrication-related constraints for machine-fabricated dovetail joints. In order to integrate the joint fabrication directly with the panel formatting, we use a side-cutting technique (Koch 1964), which is limited to a tool inclination $\beta_{\max }$. We obtain this limit from the specific geometry of the tool, tool-holder and spindle used for the joint fabrication (Fig. 5).

The parts can be assembled in two ways, as shown in Fig. 5, which allows to address a larger range of interior fold angles $\varphi$.

$$
\varphi=\arccos \frac{\left(\overrightarrow{n_{0}} \times \overrightarrow{p q}\right) \cdot\left(\overrightarrow{n_{1}} \times \overrightarrow{p q}\right)}{\left\|\left(\overrightarrow{n_{0}} \times \overrightarrow{p q}\right)\right\| \cdot\left\|\left(\vec{n}_{1} \times \overrightarrow{p q}\right)\right\|}
$$

From this we obtain the fabrication-constrained most acute fold $\varphi_{\min }=90^{\circ}-\beta_{\max }$ and most obtuse fold $\varphi_{\max }=90^{\circ}+\beta_{\max }$. With standard routing tools, this technique allows for the jointing of acute folds up to $\varphi=50^{\circ}$, which is ideal for folded plate structures. Very obtuse fold angles $\varphi \geq 140^{\circ}$, which might be required for smalltiled geodesic dome structures, cannot be fabricated with this method.
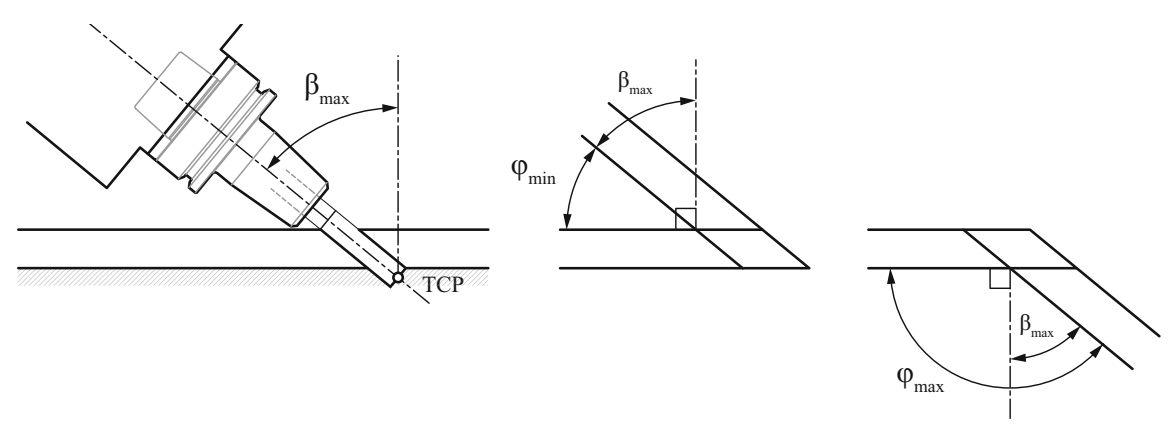

Fig. 5 Fabrication Constraints. Side-cutting technique used for the automated fabrication of 1DOF edgewise joints with common 5-axis CNC routers. The maximum tool inclination $\beta_{\max }$ results from the tool and the tool holder geometry. From this we obtain the range of possible fold angles $\pm \varphi$ between panels 


\subsection{Simultaneous Assembly of Multiple Edges}

The assembly of doubly-corrugated folded plates requires the simultaneous jointing of multiple edges per component (Fig. 6), which has implications on both the shell and the joint geometry.

For multiple 1DOF-jointed edges $E$, simultaneous assembly is only possible if the individual assembly directions $\vec{v}$ are parallel. With a normal dovetail joint geometry, where $\vec{v}=\overrightarrow{n_{0}} \times \overrightarrow{p q}$ (Fig. $2 \mathrm{~d}$, e), this is not the case: A simultaneous assembly is only possible for parallel edges, which allows only for rectangular assemblies, such as drawers or a cabinets.

In order to simultaneously join non-parallel edges, we must rotate the assembly direction $v$ of the joints to make them parallel. This possibility is known from Japanese cabinetmaking (Tetsuya 2007), where certain joints, like the Nejiri Arigata Joint are assembled along the external bisector of the fold.

We extend the Japanese technique to a cardan rotation of the frames $\left\{u_{1}, u_{2}, u_{3}\right\}$ about $u_{1}$ at the angle $\theta_{1}$ and about $u_{2}$ at the angle $\theta_{2}$ (Fig. 7a). Each of these rotations is constrained to a maximum value. $\theta_{1, \max }$ is set by the fold interior angle $180^{\circ}-\varphi_{i}$. The range is large for acute fold angles and small for obtuse fold angles. $\theta_{2, \max }$ is fabrication-constrained through the maximum tool inclination $\beta_{\max }$ (Fig. 5). The specific limit relates to $\varphi$ and $\theta_{3}$. For our setup, $\theta_{2, \max }$ was approximately $\pm 20^{\circ}$. From these constraints, we obtain a pyramid-shaped window $S$ for every edge $E$ (Fig. 7b). This window illustrates all possible assembly directions for $E$.

For the simultaneous jointing of three edges $E_{1}, E_{2}, E_{3}$, we overlay the three rotation windows $S_{1}, S_{2}, S_{3}$. If there is an intersection $S_{1} \bigcap S_{2} \bigcap S_{3}$ the edges can be jointed simultaneously. The assembly direction must be chosen within the intersection. As a result of these limited rotations, the angle between neighbouring, simultaneously jointed edges cannot be very acute. Folded plate patterns like the Herringbone, the Diamond, or the Hexagon pattern, which we chose for our prototypes, (Buri 2010) (Fig.6) work well for our joining technique. Another
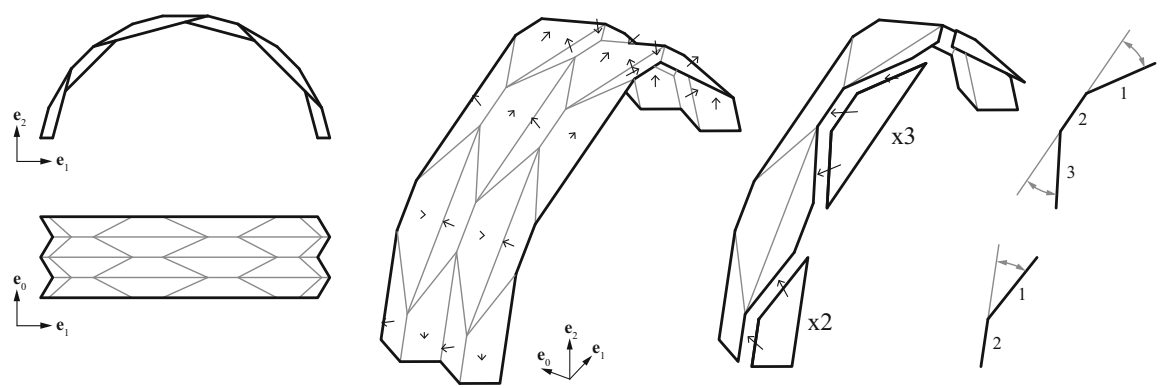

Fig. 6 The assembly of a folded plate from discrete elements (left side) requires the simultaneous assembly of non-parallel edges. (right side) We rotate the insertion direction of our 1DOF joints, to make the insertion vectors of simultaneously jointed edges parallel. We chose a hexagon reverse fold pattern which requires only moderate rotations 


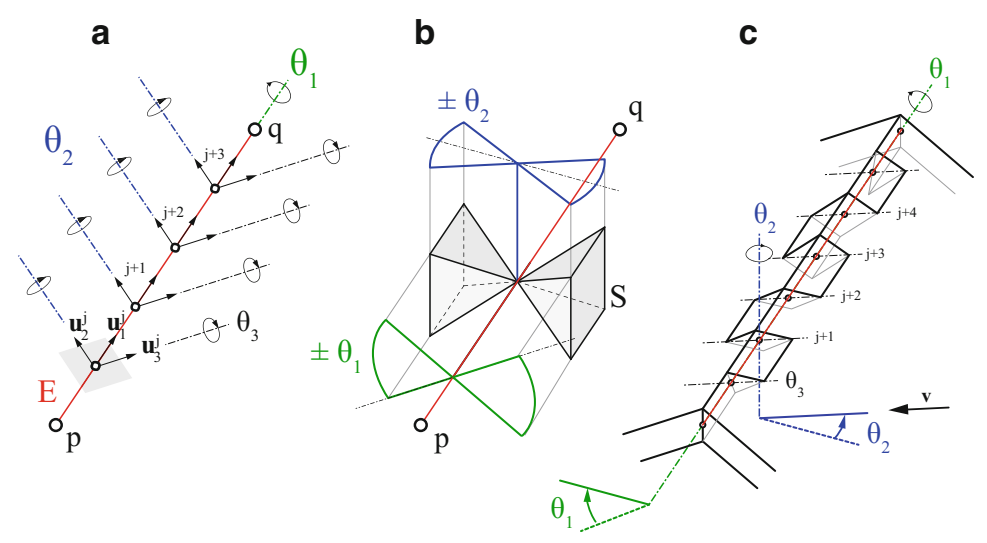

Fig. 7 (a) Rotation of the frames $\left\{u_{1}, u_{2}, u_{3}\right\}$ about the three Euler angles $\theta_{1}$ (green), $\theta_{2}$ (blue), and $\theta_{3}$. (b) Rotation window $S$, illustrating all possible assembly directions. (c) Dovetail joint with assembly direction rotated about $\theta_{1}=20^{\circ}$ and $\theta_{2}=20^{\circ}$

essential feature provided by these reverse-folds are the acute fold angles, which easily satisfies the fabrication-constrained range of $\varphi_{\min }=50^{\circ}$ to $\varphi_{\max }=140^{\circ}$.

\section{Interlocking Arch Prototype}

In an assembly of multiple components (Fig. 8), a step-by-step sequence must be planned for the assembly of the parts. The completed structure can only be disassembled piecewise in the reverse order of assembly. In this way, the elements interlock with one another like a burr puzzle (Wyatt 1928).

Each joint consists of two parts, which must be parallel during assembly. We therefore chose a folded plate geometry with relatively short edges. The manual assembly of long edges may be more difficult but can be simplified with a modified joint geometry. It is important to know the approximate direction of insertion for each part, as this is not easily visible through the joint geometry. Deformations of the arch during the assembly should be minimised. We have assembled this first prototype lying on the side. However larger assembly may require temporary punctual supports. Although the in-plane dimensional stability of the Kerto-Q panels is very high, panels may be slightly warped and some force may be necessary during assembly. While we have simply used a rubber hammer, more advanced techniques could be applied.

To understand the mechanical behaviour of the built prototype, we have applied a vertical load at mid-span of the arch and measured the vertical deflection at the same point. The total load of $821 \mathrm{~N}$ was applied in two identical load cycles consisting of four loading/unloading sub-cycles. First, a vertical load of $117 \mathrm{~N}$ was applied in seven steps, after which the load of the last four steps was removed. The loading 

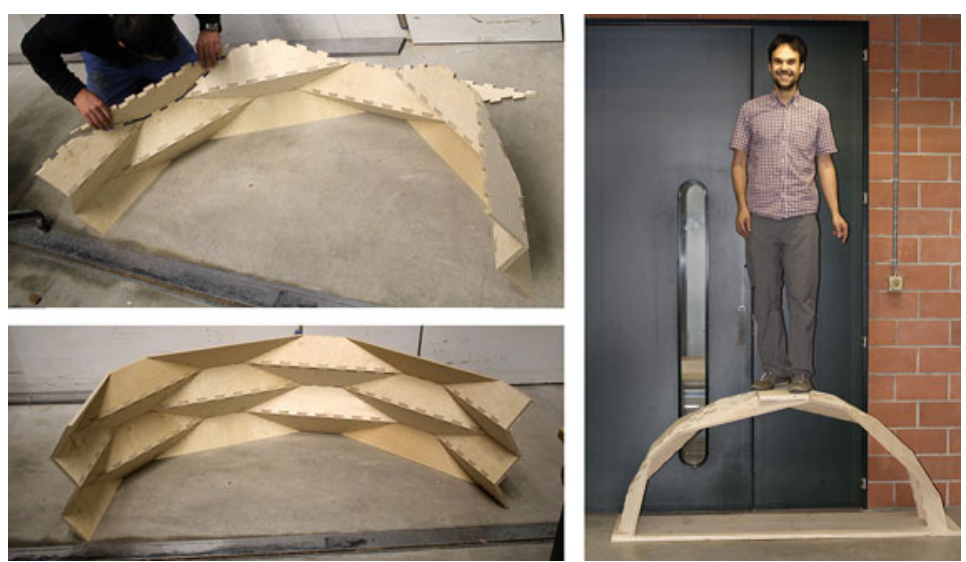

Fig. 8 Folded-plate arch prototype built from $12 \mathrm{~mm}$ birch plywood (9-layer, I-I-I-I-I). Assembled without adhesive bonding or metal fasteners. Span $1.65 \mathrm{~m}$, self-weight $9.8 \mathrm{~kg}$

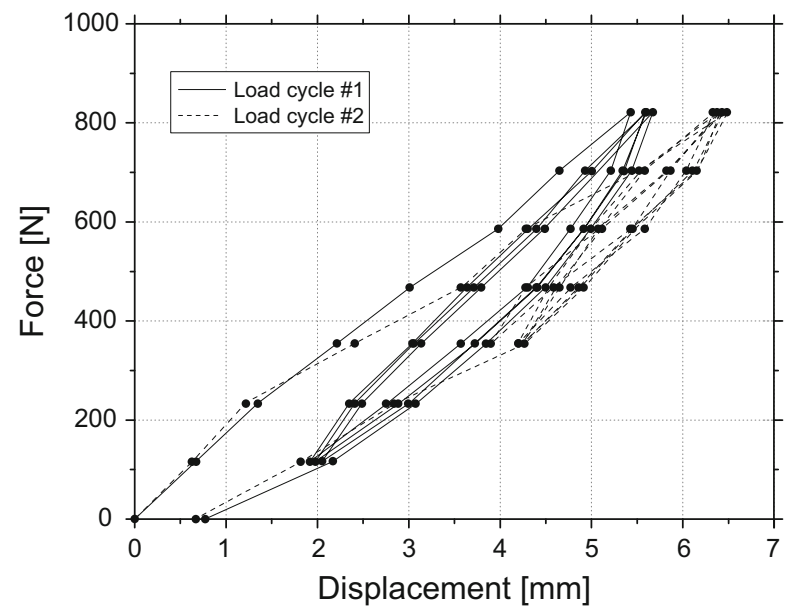

Fig. 9 Series of 3-point flexural tests on the small scale interlocking arch prototype built from Metsawood $12 \mathrm{~mm}$ birch plywood panels

and unloading of the last four steps was repeated three more times, after which the complete load was removed and the residual deflection was measured (Fig. 9).

Under a vertical load equal to the arch's dead weight of $9.8 \mathrm{~kg}(98 \mathrm{~N})$, the deflection measured at mid-span was $2 \mathrm{~mm}$. From this we obtain a span-todeflection-ratio of $L / 750$ and the arch's structural efficiency which reaches 8.6 when loaded with $821 \mathrm{~N}$ (ratio of the maximal load over the dead weight of the arch). 


\section{Interlocking Shell Prototype}

\subsection{Automatic Geometry Processing}

Using the RhinoPython application programming interface, we have developed a computational tool which lets us instantly generate both the geometry of the individual components and the machine G-Code required for fabrication. The tool processes arbitrary polygon meshes, and generates 1DOF joints for all nonnaked edges where the fold angle $\varphi$ is larger than $\varphi_{\min }$ and smaller than $\varphi_{\max }$ shown in Fig. 5 (non-smooth meshes). It also requires an input of edge identifier tuples identifying those edges which must be jointed simultaneously, as well as the thickness of the LVL panels. Exploiting this geometrical freedom, we have tested our computational tool on the design of a folded plate shell prototype with an alternating convex-concave transversal curvature. The shell spans over $3 \mathrm{~m}$ at a thickness of $21 \mathrm{~mm}$, using Kerto-Q structural grade LVL panels (7-layer, I-III-I) (Fig. 10).

Comparing this doubly-curved folded plate with a straight extrusion (as tested by Buri 2010), it can be concluded that the slight double-curvature proves to be very beneficial when it comes to global deflections, for example those caused by wind loads. Deflections for the doubly-curved shell geometry in the vertical direction are up to $39 \%$ smaller and up to $13 \%$ smaller in the lateral direction than the ones for the straight extrusion one.

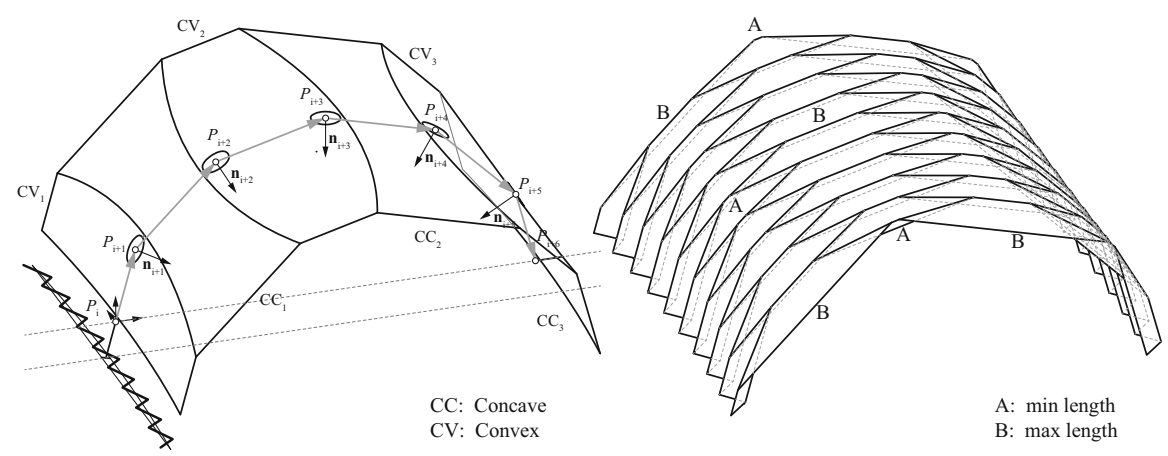

Fig. 10 Doubly-curved folded-plate: The radius $(R=17 \mathrm{~m})$ of the transversal curvature is determined by the folded plates maximum amplitude $h$ (Buri 2010), which is inversely proportional to the number of segments $m$ of the cross-section polyline (grey). We obtain this polyline from a circular arc divided into segments of equal length. The interior angle $\gamma=((m-2) * 180)-k$ of this polyline is proportional to all fold angles $\varphi$. The geometry of our prototype was fabricationconstrained to a maximum component length $B \leq 2.5 \mathrm{~m}$ 


\subsection{Assembly}

Figure 11 shows a part of the connectivity graph of the doubly-curved folded plate shell prototype. It illustrates the hierarchy, direction and order in which the 107 components with their 239 edgewise 1DOF joints must be assembled.

Figure 12 shows the components from Fig. 11 in 3D, demonstrating how the component based on mesh face $F_{86}$ is being inserted. Its three edgewise joints $E_{41}$, $E_{68}$ and $E_{89}$ must be assembled simultaneously. The three assembly vectors of the edges $\overrightarrow{v_{41}}, \overrightarrow{v_{68}}$ and $\overrightarrow{v_{89}}$ have been rotated to be parallel. The same applies for the adjacent edges on the left side of the faces $F_{67}, F_{69}, F_{88}, F_{103}$ and $F_{105}$ (see Fig. 11: all faces with multiple outgoing arrows). The roman numerals on top of Fig. 11 show the interlocking sequence. All joints marked with I. $\left(E_{18}, E_{99}, E_{100}, E_{44}\right.$, $\left.E_{66}, E_{91}, E_{59}, E_{43}\right)$ can be assembled individually and independently in a first step. Within the rotation window of the edge, we can freely rotate $\vec{v}$ for these edges (the greater the angle between $\vec{v}$ and the main direction of traction $e_{1}$, the better). After the connection of the joints marked II. $\left(E_{35}, E_{65}, E_{98}\right)$, all connections left of this point cannot be disassembled any more without disconnecting II. The components interlock with one another, similar to a burr puzzle (Wyatt 1928). The rest of the assembly follows the same logic.

\subsection{Completed Shell Prototype and Load Test}

Figure 13 shows the completed folded plate prototype, with a span of $3 \mathrm{~m}$ and a shell thickness of $21 \mathrm{~mm}$. Boundary conditions that restrain displacements of the supports in every direction, but allow rotations, were applied on both sides. A longitudinal line load was introduced along the top of the shell and vertical displacement was measured at center point (Fig. 14).

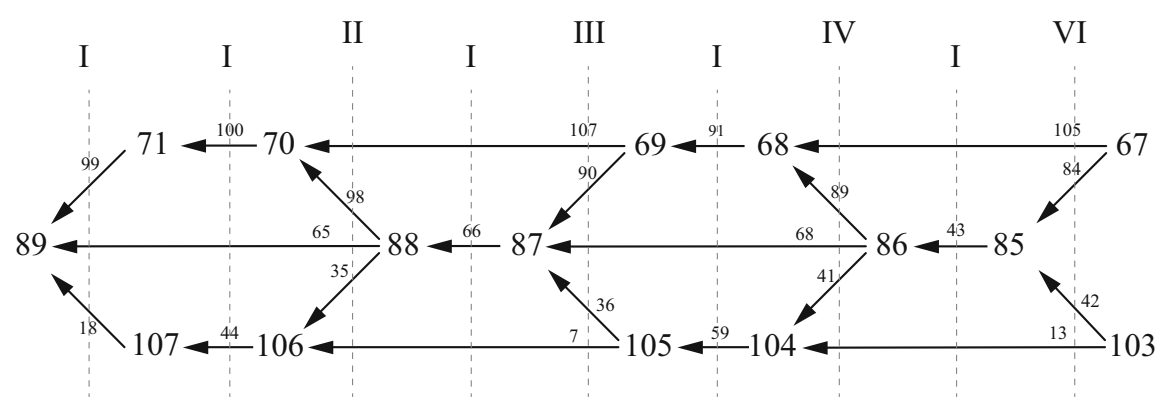

Fig. 11 Partial connectivity graph of the folded plate shell prototype. (Left-to-right side assembly) Large numbers represent mesh Faces $F$, small numbers represent mesh edges $E$. Roman numerals illustrate the interlocking hierarchy 


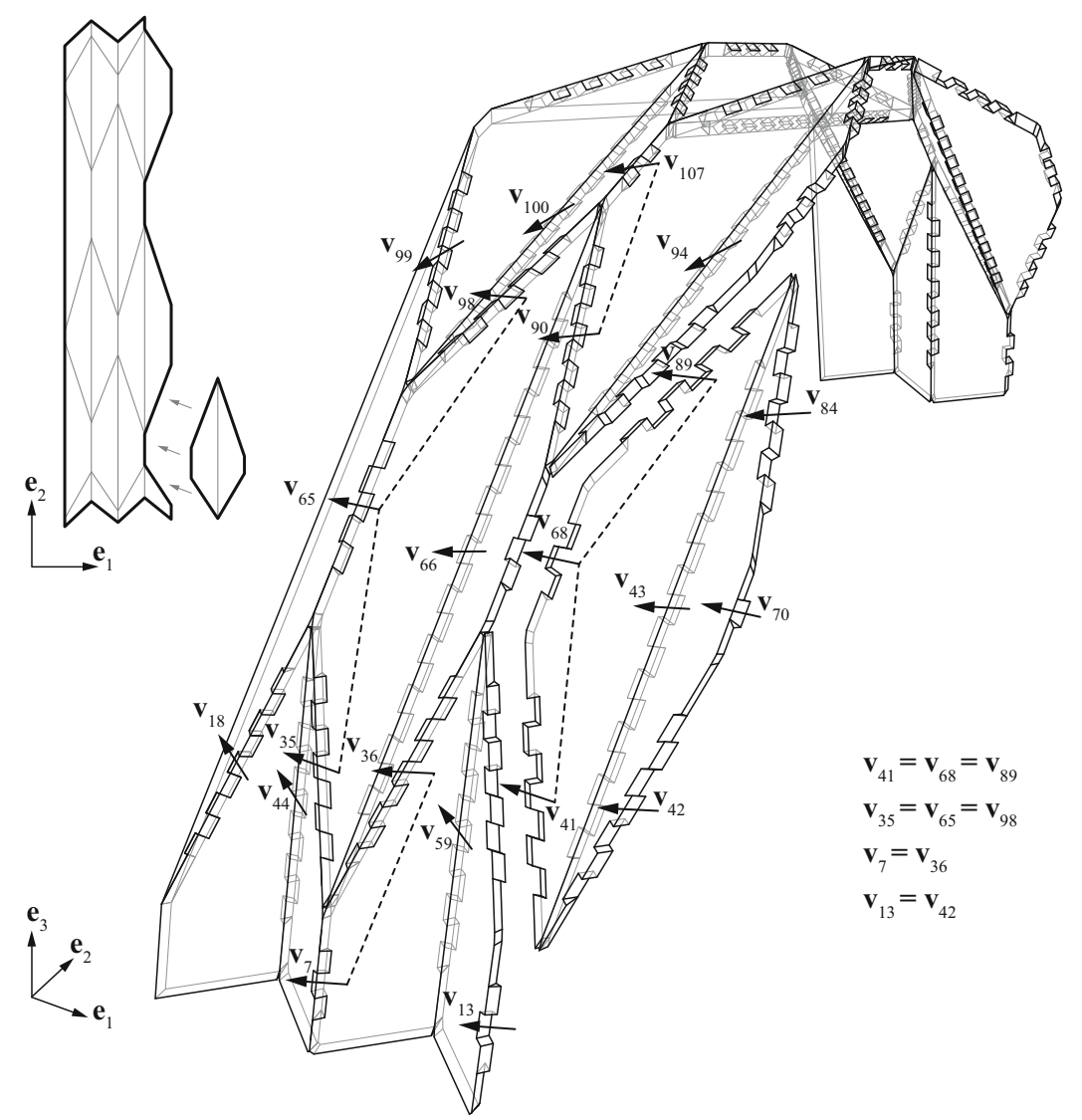

Fig. 12 Left-to-right-side assembly of the Interlocking folded plate shell prototype. Built from Kerto-Q structural grade LVL panels (7-layer, I-III-I)

The prototype structure was also modelled in FE analysis software (Abaqus) and loaded in the same way. The plates were modelled using shell elements, where the mid-surface is used to represent the 3D plate and transverse shearing strains are neglected. Connections between the plates were considered as completely rigid in order to obtain minimal displacements of the structure. By comparing the displacements of the structure with infinitely stiff joints with the ones measured on the prototype, we obtained information about the actual semi-rigidity of the joints. The results obtained from the testing of the large scale prototype showed that the load of $25 \mathrm{kN}$, that corresponds to the proportional limit of the load-displacement curve, causes a vertical displacement of $23 \mathrm{~mm}$. In the FE model, the load applied in the same manner caused a vertical displacement of $2.6 \mathrm{~mm}$. 


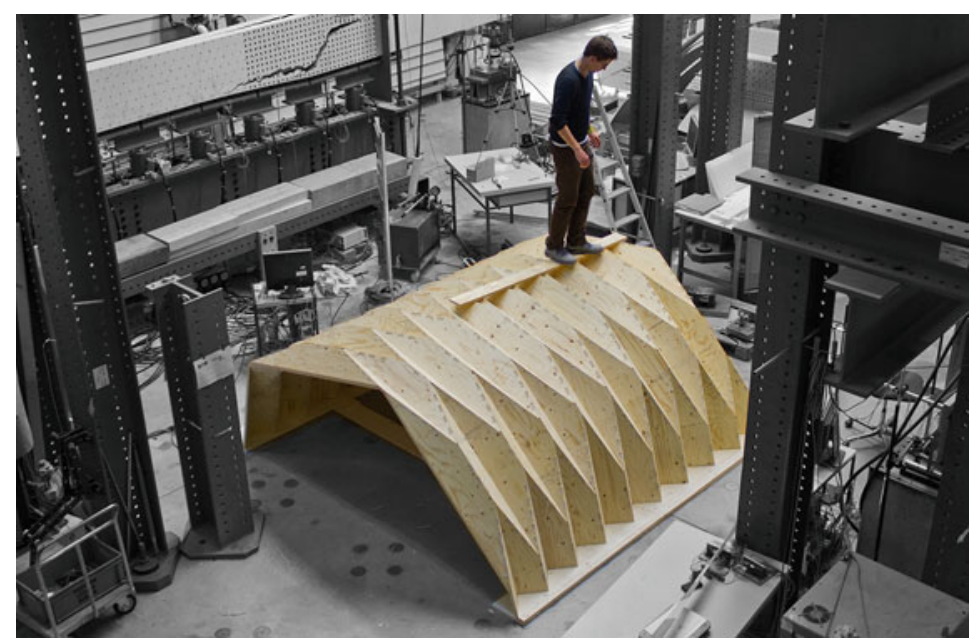

Fig. 13 Folded-plate shell prototype, built from $21 \mathrm{~mm} \mathrm{LVL}$ panels. With a self-weight of $192 \mathrm{~kg}$, the prototype with a span of $3 \mathrm{~m}$ was tested with a line-load up to $45 \mathrm{kN}$

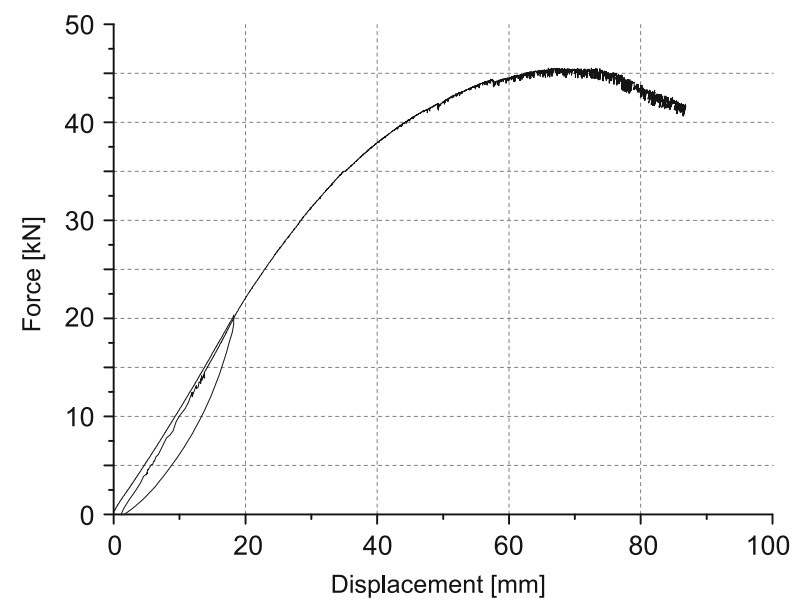

Fig. 14 Load-displacement curve of the shell prototype. A longitudinal line load was introduced along the top of the shell. Vertical displacement was measured at the center point

\section{Conclusion}

A timber folded plate shell combines the structural advantages of timber panels with the efficiency of folded plates. However, in such discrete element assemblies, a large amount of semi-rigid joints must provide sufficient support

(continued) 
for the adjacent plates in order to ensure an efficient load-bearing system. This remains a challenge with much potential for improvements (Hahn 2009).

Integrated edgewise joints present an interesting addition and an alternative to state-of-the-art connectors: Compared to adhesive bonding, such joints can be assembled rapidly on site. Also, compared to costly metal plates and fasteners, which are typically required in large quantities (Neuhaus 2004), the fabrication of integrated joints is not more expensive. The replacement or reduction of metal fasteners with an integrated mono-material connection includes advantages such as improved aesthetics, ease-of recycling or a homogenous thermal conductivity of the parts, which can reduce condensation and decay. Graubner (1986) Another particular advantage is the possibility to join thin panels: The current technical approval for the Kerto-Q panels does not permit screwed joints on panels with a thickness of less than $60 \mathrm{~mm}$ (DIBt 2011).

Recent experimental projects have already demonstrated first applications of integrated edgewise joints for timber panels. This paper followed up on these projects, examining the particular advantages, potential and challenges of 1DOF joints for timber folded plate shells. We have demonstrated how this joint geometry helps resisting the forces which occur in such structures. In addition to the load-bearing connector features, the joints provide locator features, which allow for precise positioning and alignment of the parts through the joint geometry. This improves both accuracy and ease of assembly. Furthermore, we have presented a solution for the simultaneous assembly of multiple edges per panel, which is essential for the application of 1DOF joints in a folded plate shell structure. The per edge "rotation window" integrates the joint constraints related to assembly and fabrication. It can be processed algorithmically and give instant feedback on wether or not a set of non-parallel edges can be jointed simultaneously. This provides a tool for the exploration of a variety of alternative folded plate shell geometries.

The prototypes presented in this paper already suggest possible patterns and demonstrate the reciprocal relationship between the geometry of the plates and the joints. Two built structures allowed us to test and verify the proposed methods for fabrication and assembly while providing valuable information about the load-bearing capacity of the integrated joints.

For the application in a large-scale building structure, further research is required to determine if the integrated joints can replace additional connectors entirely or reduce their amount. A possible combination of integrated joints with additional metal fasteners has been demonstrated recently in the LaGa Exhibition Hall (ICD/ITKE 2014). Another possibility would be a combination of the $1 \mathrm{DOF}$ joints with integrated elastic interlocks (Robeller et al. 2014a; Simek and Sebera 2010) 
Acknowledgements We would like to thank Stéphane Nicolas Roche for the discussions, as well as Gabriel Tschanz for assisting with the fabrication and assembly of the prototypes. We also thank Jouni Hakkarainen and the Metsa Group for the supply of information and materials.

\section{References}

Buri, H.: Origami - folded plate structures. Doctoral thesis, EPFL, Lausanne (2010)

DIBt: Allgemeine bauaufsichtliche Zulassung Kerto-Q Z-9.1-100, Paragraph 4.2 and Attachment No 7, Table 5. Deutsches Institut für Bautechnik (2011)

Graubner, W.: Holzverbindungen,Gegenüberstellung von Holzverbindungen Holz in Holz und mit Metallteilen. Deutsche Verlags-Anstalt Stuttgart (1986)

Hahn, B.: Analyse und Beschreibung eines räumlichen Tragwerks aus Massivholzplatten. Master thesis, EPFL, Lausanne (2009)

HESS: Hess limitless (2014). http://www.hess-timber.com/de/produkte/

Hundegger: Hundegger (2014). http://www.hundegger.de/en/machine-building/company/ourhistory.html

ICD/ITKE: Laga exhibition hall (2014). http://icd.uni-stuttgart.de/?p=11173

Koch, P.: Wood Machining Processes. Wood Processing. Ronald Press, New York (1964)

Krieg, O., et al.: Hygroskin: Meteorosensitive pavilion. In: Fabricate 2014 Conference, Zurich, pp. 272-279 (2014)

La Magna, R., et al.: From nature to fabrication: biomimetic design principles for the production of complex spatial structures. Int. J. Spat. Struct. 28, 27-40 (2013)

Messler, R.W.: Integral Mechanical Attachment: A Resurgence of the Oldest Method of Joining. Butterworth Heinemann, Burlington (2006)

Neuhaus, H.: DIN EN 1995 (Eurocode 5) - design of timber structures. DIN Deutsches Institut fur Normung e. V. (2004)

Pizzi, E.: Renzo Piano. Birkhaeuser, Basel (2003)

Purbond: National Technical Approval Z-9.1-711/Single-component polyurethane adhesive for the manufacture of engineered wood products. DiBT. based on the test results of the Otto-GrafInstitute (MPA, University Stuttgart) (2011)

Robeller, C., Mayencourt, P., Weinand, Y.: Snap-fit joints: integrated mechanical attachment of structural timber panels. In: Proceedings of the 34th International Conference of the Association of Computer-aided Design in Architecture ACADIA, Los Angeles (2014a)

Robeller, C., Nabaei, S.S., Weinand, Y.: Design and fabrication or robot-manufactured joints for a curved-folded thin-shell structure made from CLT. In: Robotic Fabrication in Architecture, Art and Design 2014, pp. 67-81. Springer, Dordrecht (2014b)

Sebera, V., Simek, M.: Finite element analysis of dovetail joint made with the use of CNC technology. Acta Univ. Agric. Silvic. Mendel. Brun. 58, 321-328 (2010)

Simek, M., Sebera, V.: Snap-fit joints: integrated mechanical attachment for structural timber panels. In: Proceedings of the International Convention of Society of Wood Science and Technology and United Nations Economic Commission for Europe, Geneva (2010)

Tetsuya, K.: Dento kanehozo kumitsugi : Sekkei to seisaku no jissai. LLP Gijutsushi Shuppankai, Seiunsha, Tokyo (2007)

Trautz, M., Herkrath, R.: The application of folded plate principles on spatial structures with regular, irregular and free-form geometries. In: Proceedings of the International Association for Shell and Spatial Structures (IASS) Symposium 2009, Valencia (2009)

Wyatt, E.: Puzzles in Wood. Bruce Publishing, Milwaukee (1928) 